\title{
Cosine-modulated Non-uniform Filter Banks
}

\author{
F. Argenti $\quad$ B. Brogelli $\quad$ E. Del Re \\ Dipartimento di Ingegneria Elettronica, Università di Firenze \\ Via di Santa Marta, 3 - 50139 Firenze - Italy \\ Tel.: +3955 4796 274 - Fax: +3955 4796485 \\ e-mail: argentiocosimo.ing.unifi.it
}

\begin{abstract}
In this work a method to design filter banks that allow to split the spectrum of a signal into non-uniform width subbands is presented. The filters of the banks are obtained by the cosine modulation of more than one prototype. The prototypes are designed by imposing the cancellation of the main component of aliasing in the reconstructed signal. Aliasing components and their cancellation in rational sampling factors banks are described in the details and design examples are presented.
\end{abstract}

\section{INTRODUCTION}

In this work the problem of designing filter banks that analyze the input signal into non-uniform width subbands is addressed. Non-uniform filter banks have been studied, for example, in [1]-[6]. Here, a new method to design filter banks with rational decimation factors is proposed: it extends some preliminary work, presented in [7], which considered only integer decimation factors. The design method is based on the cosine modulation of different linear phase prototypes; it does not use complex prototypes as in [6] and all the filters are obtained through a direct design, i.e., the filters of the banks are not designed as a combination of narrower passband filters. The design aims at the cancellation of the main component of aliasing in the reconstructed signal: this imposes constraints on the prototypes which become related to each other. Experimental results to show the effectiveness of the procedure are also shown.

\section{ALIASING COMPONENTS IN COSINE-MODULATED} NON-UNIFORM FILTER BANKS

Consider the system in Fig. 1, where non-uniform analysis/synthesis banks having rational sampling factors $R_{m} / M_{m}, m=0, \ldots, M-1$, are shown. The input-output relationship in the $z$-domain is given by:

$$
\begin{aligned}
\hat{X}(z) & =\sum_{m=0}^{M-1} \frac{1}{R_{m}} \frac{1}{M_{m}} \sum_{p=0}^{R_{m}-1} F_{m}\left(z^{\frac{1}{R_{m}}} W_{R_{m}}^{p}\right) \\
& \cdot H_{m}\left(z^{\frac{1}{R_{m}}} W_{R_{m}}^{p}\right) X(z)+ \\
& +\sum_{m=0}^{M-1} \frac{1}{R_{m}} \frac{1}{M_{m}} \sum_{l=1}^{M_{m}-1} \sum_{p=0}^{R_{m}-1} F_{m}\left(z^{\frac{1}{R_{m}}} W_{R_{m}}^{p}\right) \\
& \cdot H_{m}\left(z^{\frac{1}{R_{m}}} W_{R_{m}}^{p} W_{M_{m}}^{l}\right) X\left(z W_{M_{m}}^{l R_{m}}\right)
\end{aligned}
$$

where $W_{M}=e^{-j 2 \pi / M}$. Eq. (1) highlights the reconstruction transfer function and the aliasing terms. Suppose that each filter has real coefficients and is obtained through the cosine-modulation of a low-pass prototype; therefore, the impulse responses of the $m$-th branch analysis/synthesis filters are given by:

$$
\begin{aligned}
h_{m}(n) & =2 g_{m}(n) \cos \left(\left(2 k_{m}+1\right) \frac{\pi}{2 M_{m}}\left(n-\frac{N_{m}-1}{N^{2}}\right)+\theta_{m}\right) \\
f_{m}(n) & =2 g_{m}(n) \cos \left(\left(2 k_{m}+1\right) \frac{\pi}{2 M_{m}}\left(n-\frac{N_{m}-1}{2}\right)-\theta_{m}\right) \\
& =h_{m}\left(N_{m}-1-n\right)
\end{aligned}
$$

for $m=0,1, \ldots, M-1 . N_{m}$ is the length of $g_{m}(n)$. The prototypes $g_{m}(n)$ have a linear phase and satisfy $g_{m}(n)=g_{m}\left(N_{m}-1-n\right)$. The index $k_{m}$ selects where the passband is located, while the phase terms $\theta_{m}$ are chosen to satisfy the aliasing cancellation constraint, as shown in the following.

If we translate (2) in the $z$-domain, then the transfer functions of each filter can be expressed as:

$$
\begin{aligned}
& H_{m}(z)=U_{m}(z)+V_{m}(z) \\
& F_{m}(z)=\hat{U}_{m}(z)+\hat{V}_{m}(z)
\end{aligned}
$$

where

$$
\begin{array}{ll}
U_{m}(z)= & G_{m}\left(z W_{2 M_{m}}^{\left(k_{m}+(1 / 2)\right)}\right) W_{2 M_{m}}^{\left(k_{m}+(1 / 2)\right)\left(N_{m}-1\right) / 2} \\
& \cdot e^{j \theta_{m}} \\
V_{m}(z)= & G_{m}\left(z W_{2 M_{m}}^{-\left(k_{m}+(1 / 2)\right)}\right) W_{2 M_{m}}^{-\left(k_{m}+(1 / 2)\right)\left(N_{m}-1\right) / 2} \\
& \cdot e^{-j \theta_{m}} \\
\hat{U}_{m}(z)= & G_{m}\left(z W_{2 M_{m}}^{\left(k_{m}+(1 / 2)\right)}\right) W_{2 M_{m}}^{\left(k_{m}+(1 / 2)\right)\left(N_{m}-1\right) / 2} \\
& \cdot e^{-j \theta_{m}} \\
\hat{V}_{m}(z)= & G_{m}\left(z W_{2 M_{m}}^{-\left(k_{m}+(1 / 2)\right)}\right) W_{2 M_{m}}^{-\left(k_{m}+(1 / 2)\right)\left(N_{m}-1\right) / 2} \\
& \cdot e^{j \theta_{m}}
\end{array}
$$

As can be easily verified, $U_{m}(\omega)$ and $\hat{U}_{m}(\omega)$ are rightshifted versions of $G_{m}(\omega)$, while $V_{m}(\omega)$ and $\hat{V}_{m}(\omega)$ are left-shifted versions of $G_{m}(\omega)$.

Due to the $M_{m}$-fold upsampler in the synthesis stage, images of the $m$-th subband spectrum are filtered by $F_{m}(z)$. The main aliasing components are produced at the low-frequency and at the high-frequency edges of the passband of $F_{m}(z)$ : let $A_{m}^{(l o w)}(z)$ and $A_{m}^{(h i g h)}(z)$ be these components, respectively. They have been described for an uniform bank in [8]. If we consider that each branch operates on an $R_{m}$-fold upsampled version of $x(n)$ and if only the more relevant terms are retained, then the main component of aliasing can be expressed 
as:

$$
\begin{aligned}
& A_{m}^{(\text {low })}(z)= \\
& \frac{1}{M_{m}}\left[\hat{U}_{m}(z) V_{m}\left(z W_{M_{m}}^{k_{m}}\right) X\left(z^{R_{m}} W_{M_{m}}^{k_{m} R_{m}}\right)+\right. \\
& \left.+\hat{V}_{m}(z) U_{m}\left(z W_{M_{m}}^{-k_{m}}\right) X\left(z^{R_{m}} W_{M_{m}}^{-k_{m} R_{m}}\right)\right] \\
& \frac{1}{M_{m}}\left[\hat{U}_{m}(z) V_{m}\left(z W_{M_{m}}^{\left(k_{m}+1\right)}\right) X\left(z^{R_{m}} W_{M_{m}}^{\left(k_{m}+1\right) R_{m}}\right)+\right. \\
& \left.+\hat{V}_{m}(z) U_{m}\left(z W_{M_{m}}^{-\left(k_{m}+1\right)}\right) X\left(z^{R_{m}} W_{M_{m}}^{\left(k_{m}+1\right) R_{m}}\right)\right](8)
\end{aligned}
$$$$
A_{m}^{(h \dot{s} g)}(z)=
$$

In [8] it is shown that for uniform cosine-modulated filter banks the component $A_{m}^{(h i g h)}(z)$ of the $m$-th branch is cancelled by the component $A_{m+1}^{(\text {low })}(z)$ of the $(m+1)$ th branch. In the non-uniform case, we have to consider that the cancellation may occurr also by coupling the (high)-(high), (low)-(low) or (low)-(high) aliasing components coming from the $m$-th and from the $(m+1)$ th branch. For example, consider the bank $\{1 / 5,3 / 5$, $1 / 5\}$ that can be implemented using filters having a passband equal to $\pi / 5$ and centered, on the positive frequency axis, in $\pi / 10, \pi / 2$ and $9 \pi / 10$. Two different prototypes are necessary to design the filters in the $m=0$ and $m=1$ branches; moreover, we have to choose $k_{0}=0$ and $k_{1}=2$. The aliasing component $A_{0}^{(h i g h)}(z)$ produced in the $m=0$ branch at the synthesis stage must be cancelled by $A_{1}^{(h i g h)}(z) \downarrow 3$, where $Q(z) \downarrow M$ denotes the $z$-transform of the $M$-fold subsampled version of $q(n)$.

In the following section the constraints that the aliasing cancellation imposes on the prototypes are discussed.

\section{ALIASING CANCELLATION AND DESIGN PROCEDURE}

In general, four cases must be taken into account:
a) $A_{m}^{(h i g h)}(z) \downarrow R_{m}+A_{m+1}^{(h i g h)}(z) \downarrow R_{m+1}=0$
b) $A_{m}^{(\text {low })}(z) \downarrow R_{m}+A_{m+1}^{(\text {low })}(z) \downarrow R_{m+1}=0$
c) $A_{m}^{(\text {low })}(z) \downarrow R_{m}+A_{m+1}^{(\text {high })}(z) \downarrow R_{m+1}=0$
d) $A_{m}^{(h i g h)}(z) \downarrow R_{m}+A_{m+1}^{(l o w)}(z) \downarrow R_{m+1}=0$

Consider the case $a$ ). In [9] it is shown that if the phase terms satisfy one of the conditions shown in the first row, third column of Table 1 (the condition to be chosen depends on the values of $k_{m}, R_{m}, M_{m}, k_{m+1}$ $R_{m+1}$ and $M_{m+1}$ ) then aliasing cancellation occurs if

$$
\begin{aligned}
& \frac{1}{M_{m}} \frac{1}{R_{m}} \sum_{p=0}^{R_{m}-1} G_{m}\left(z^{1 / R_{m}} W_{R_{m}}^{p}\right) \\
& G_{m}\left(z^{1 / R_{m}} W_{R_{m}}^{p} W_{2 M_{m}}\right)=\frac{1}{M_{m+1}} \frac{1}{R_{m+1}} \sum_{p=0}^{R_{m+1}-1} \\
& G_{m+1}\left(z^{1 / R_{m+1}} W_{R_{m+1}}^{p} W_{4 M_{m}}^{R_{m} / R_{m+1}} W_{4 M_{m+1}}^{-1}\right) \\
& G_{m+1}\left(z^{1 / R_{m+1}} W_{R_{m+1}}^{p} W_{4 M_{m}}^{R_{m} / R_{m+1}} W_{4 M_{m+1}}\right)
\end{aligned}
$$

It can be demonstrated [9] that the cancellation of aliasing for the cases b)-d) leads to the same constraint on the prototypes.

When we substitute $z=e^{j \omega}$, the left hand side (right hand side) of (9) is a $2 \pi$-periodic function in $\omega$ and is composed by the sum of $R_{m}\left(R_{m+1}\right)$ periodic functions with period $2 R_{m} \pi\left(2 R_{m+1} \pi\right)$. Therefore, we have to match the main component of aliasing of each side of (9) that falls in the interval $(-\pi, \pi)$; this occurs by choosing the index $p$ of the summations equal to zero. Moreover, consider the zero-phase filters associated with each linear phase prototype. Their frequency responses are real functions and are defined as

$$
G_{m}^{(z p)}(\omega)=G_{m}(\omega) e^{j \frac{N_{m}-1}{2} \omega}
$$

If (10) and $p=0$ are substituted into (9) and if the fol lowing condition on the lengths of the prototype filters is imposed

$$
\frac{N_{m}-1}{R_{m}}=\frac{N_{m+1}-1}{R_{m+1}}
$$

then we obtain the final constraint on the frequency responses of the zero-phase prototype filters, that is

$$
\begin{aligned}
& \frac{1}{M_{m}} \frac{1}{R_{m}} G_{m}^{(z p)}\left(\frac{\omega}{R_{m}}\right) G_{m}^{(x p)}\left(\frac{\omega}{R_{m}}-\frac{\pi}{M_{m}}\right)= \\
& \frac{1}{M_{m+1}^{\prime}} \frac{1}{R_{m+1}} G_{m+1}^{(z p)}\left(\frac{\omega}{R_{m+1}}-\frac{\pi R_{m}}{2 M_{m} R_{m+1}}+\frac{\pi}{2 M_{m+1}}\right) \\
& G_{m+1}^{(z p)}\left(\frac{\omega}{R_{m+1}}-\frac{\pi R_{m}}{2 M_{m} R_{m+1}}-\frac{\pi}{2 M_{m+1}}\right)
\end{aligned}
$$

Suppose that the prototypes have an ideal stopband, i.e., with zero gain; hence (12) can be fulfilled by a proper choice of the transition band. The transition bandwidth of $G_{m+1}(\omega)$ must be $R_{m} / R_{m+1}$ times that of $G_{m}(\omega)$, while the magnitudes will have to be proportional according to the factor $\sqrt{\frac{R_{m} M_{m}}{R_{m+1} M_{m+1}}}$. Let $\omega_{c, m}=\pi /\left(2 M_{m}\right)$ be the cut-off frequency of $G_{m}(\omega)$. Suppose the transition band, having width $\Delta \omega_{m}$, is centered in $\omega_{c, m}$ and let $\omega_{p, m}=\omega_{c, m}-\left(\Delta \omega_{m} / 2\right)$ and $\omega_{s, m}=\omega_{c, m}+\left(\Delta \omega_{m} / 2\right)$ be the upper bound of the passband and the lower bound of the stopband, respectively, of $G_{m}(\omega)$. Therefore, the transition bands of the prototypes must satisfy $R_{m} \Delta \omega_{m}=R_{m+1} \Delta \omega_{m+1}$. Moreover, (12) is satisfied if the zero-phase frequency response of the prototype $G_{m+1}(\omega)$ is derived as follows:

$$
\begin{aligned}
& G_{m+1}^{(z p)}(\omega)= \\
& =\left\{\begin{array}{lr}
0 & -\pi<\omega \leq-\omega_{s, m+1} \\
\sqrt{\frac{R_{m+1} M_{m+1}}{R_{m} M_{m}}} G_{m}^{(z p)}\left(-\omega_{p, m}+\left(\omega+\omega_{p, m+1}\right)\right. \\
\left.\frac{\sqrt{\omega_{s, m}-\omega_{p, m}}}{\omega_{m+1}-\omega_{p, m+1}}\right) & -\omega_{s, m+1}<\omega \leq-\omega_{p, m+1} \\
\sqrt{\frac{R_{m+1} M_{m+1}}{R_{m+1} M_{m+1}}} & -\omega_{p, m+1}<\omega \leq \omega_{p, m+1} \\
\left.\frac{\omega_{s, m} M_{m}}{\omega_{s, m+1}-\omega_{p, m+1}}\right) & \omega_{p, m}+\left(\omega-\omega_{p, m+1}\right) \\
0 & \quad \omega_{p, m+1}<\omega \leq \omega_{s, m+1} \\
0 & \quad \omega_{s, m+1}<\omega \leq \pi
\end{array}\right.
\end{aligned}
$$


Hence, the prototype filters that lead to aliasing cancellation can not be designed independently of each other. Assuming the aliasing components have been completely eliminated, the input-output relationship shown in (1) becomes

$$
\begin{aligned}
\hat{X}(z)= & {\left[\sum_{m=0}^{M-1} \frac{1}{R_{m}} \frac{1}{M_{m}} \sum_{p=0}^{R_{m}-1} F_{m}\left(z^{\frac{1}{R_{m}}} W_{R_{m}}^{p}\right)\right.} \\
& \left.\cdot H_{m}\left(z^{\frac{1}{R_{m}}} W_{R_{m}}^{p}\right)\right] X(z)=T(z) X(z)
\end{aligned}
$$

The prototype filter lengths are constrained to satisfy the condition $\frac{N_{m}-1}{R_{m}}=c, m=0, \ldots, M-1$, where $c$ is a constant and represents the delay introduced by the analysis/synthesis banks. We will suppose that $\mathrm{c}$ is an integer, so that the signals reconstructed in each branch of the structure can be easily realigned. Phase error is absent since the synthesis filters are a time reversed version of the analysis filters, while the magnitude error is maintained at low levels if $T(z)$ is approximately allpass. The reconstruction error is reduced choosing prototype filters with high stopband attenuation and with a power complementary transition band, i.e., satisfying

$$
\begin{gathered}
\left|G_{m}(\omega)\right|^{2}+\left|G_{m}\left(\frac{\pi}{M_{m}}-\omega\right)\right|^{2}=R_{m} M_{m} \\
\text { for } \omega_{p, m}<\omega<\omega_{s, m}
\end{gathered}
$$

Prototype filters built by using (13) still maintain the power-complementary transition band property and make $T(z)$ approximately allpass.

The procedure that allows to design a filter bank with given rational decimation factors foresees the following steps:

1) Select the index $m$ for which $R_{k} / M_{k}, k=0, \ldots, M$ 1 , is minimum. Design a real coefficients prototype $g_{m}(n)$, with linear phase $G_{m}(\omega)$, cut-off frequency $\omega_{c, m}=\frac{\pi}{2 M_{m}}$, characterized by a gain $\sqrt{R_{m} M_{m}}$ in the passband and by a power complementary transition band, i.e., satisfying (15). For example, the methods described in [10][11][6] can be used.

2) Find the zero-phase filter $G_{m}^{(z p)}(\omega)$ related to $G_{m}(\omega): G_{m}^{(z p)}(\omega)$ is a real function symmetrical around $\omega=0$; build the zero-phase frequency response $G_{m+1}^{(z p)}(\omega)$ according to $(13)$ and obtain $G_{m+1}(\omega)$ by using $(10)$; compute $g_{m+1}(n)$ from $G_{m+1}(\omega)$ by truncated Inverse Fourier Transform or by using the frequency sampling method.

3) Repeat step 2) for each pair of branches of the structure that needs a new prototype filter to be designed. The non-uniform bank can be built through cosine modulation of the prototypes and by using the correct phase terms (see Table 1).

In the following section this procedure is used to design examples of non-uniform banks.

\section{EXPERIMENTAL RESULTS}

To show the effectiveness of the design procedure described in the previous section we have considered two examples of non-uniform banks. We will indicate with $\mathrm{K}$ and $\Theta$ the sets $\left\{k_{m}, m=0, \ldots, \mathrm{M}-1\right\}$ and $\left\{\theta_{m}\right.$, $m=0, \ldots, M-1\}$, respectively.

Example 1: Non-uniform bank composed by four branches with rational decimation factors $\{2 / 7,2 / 7$,
$2 / 7,1 / 7$. Two prototypes have to be designed $\left(g_{0}(n)=g_{1}(n)=g_{2}(n)\right)$. In this example more than one choice is possible for $K$. We will use $K=\{0,5,4,6\}$ to show the largest variety of couplings of aliasing components (high)-(high), (low)-(high), (low)-(low), in the order). In this case $\theta=\{\pi / 4, \pi / 4,-\pi / 4,-\pi / 4\}$.

Example 2: Non-uniform bank composed by four branches with rational decimation factors $\{1 / 2,1 / 4$, $3 / 16,1 / 16\}$. This is an example in which the rational sampling factors have different denominators. All the aliasing couplings are of the type (high)-(low); $\mathrm{K}=\{0$, $2,4,15\} ; \theta=\{\pi / 4 ;-\pi / 4, \pi / 4,-\pi / 4\}$.

The performance of the presented design method is evaluated in terms of both the overall distortion function $T(\omega)$ and the residual aliasing error. As to the latter error, a global measure relative to the whole structure is used in this work. According to the input-output relationship in (1), the aliasing contribution relative to $X\left(z W_{M_{m}}^{l R_{m}}\right)$ can be written as

$$
A_{l, m}(z)=\frac{1}{R_{m}} \frac{1}{M_{m}} \sum_{p=0}^{R_{m}-1} H_{m}\left(z^{\frac{1}{R_{m}}} W_{R_{m}}^{p} W_{M_{m}}^{l}\right)
$$

with $m=0, \ldots, M-1, l=0, \ldots, M_{m}-1$. The functions $A_{l, m}(\omega)$ are $2 \pi$-periodic functions all the aliasing terms $A_{l, m}(z)$ that refer to the same shifted version of $X(z)$, i.e., having the same value of $W_{M_{m}}^{R_{m}}$, must be summed up, so that the following aliasing error can be defined:

$$
E_{a}(\omega)=\sqrt{\sum_{r=1}^{M_{\max }-1}\left|\sum_{m=0}^{M-1} \sum_{l=1,\left(l R_{m}\right) \bmod M_{m}=r}^{M_{m}-1} A_{l, m}(\omega)\right|^{2}}
$$

where $M_{\max }=\max \left\{M_{m}, m=0, \ldots, M-1\right\}$ and where the inner summation in (17) is evaluated only for the values of $l$ and $m$ satisfying the condition $\left(l R_{m}\right) \bmod M_{m}=r$.

Therefore

$$
\begin{gathered}
E_{p-p}=\max _{0 \leq \omega \leq \pi}|T(\omega)|-\min _{0 \leq \omega \leq \pi}|T(\omega)| \\
E_{a, \max }=\max _{0 \leq \omega \leq \pi} E_{a}(\omega)
\end{gathered}
$$

can be used as measures of the quality of the designed banks.

Table 2 and 3 reports the results obtained for Example 1 and 2, respectively, for different lengths of the prototypes. As can be seen, both the magnitude distortion and the aliasing error are kept small

In Fig. 2 the frequency responses of the final cosine modulated analysis filters relative to Example 1 and obtained with prototypes having 82 and 163 coefficients are shown: from the inspection of this figure it can be deduced that the design based on (13) does not degrade the passband and the stopband characteristics of the new prototypes.

\section{CONCLUSIONS}

In this work a method to design non-uniform fiter banks with rational sampling factors has been presented. The method is simple and requires numerical optimization of only one prototype, being the others derived in a straightforward way from this one. For this reason, the method seems particularly suitable when banks with a large number of coefficients müst be designed. 


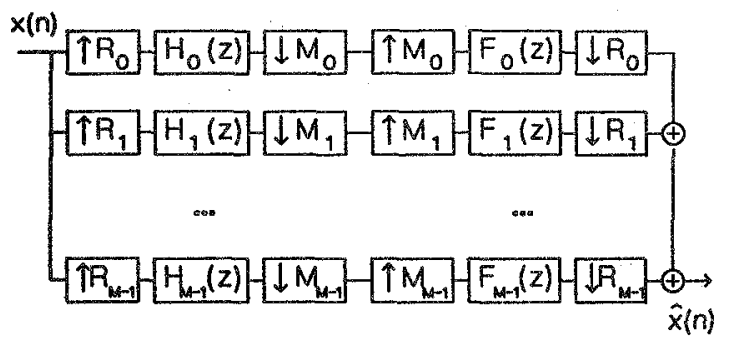

Figure 1. Rational sampling factors fifter banks

\section{REFERENCES}

[1] P.Q. Hoang and P.P. Vaidyanathan, "Non-uniform Multirate Filter Banks: Theory and Design", in Proc. Int. Symp. Circuits Syst., pp. 371-374, May 1986.

[2] K. Nayebi, T.P. Barnwell III and M.J.T. Smith, "Nonuniform Filter Banks: A Reconstruction and Design Theory", IEEE Trans. Signal Processing, Vol. 41, no. 3, pp. 1114-1127, Mar. 1993.

[3] J. Kovacevic and M. Vetterli, "Perfect Reconstruction Filter Banks with Rational Sampling Factors", IEEE Trans. Signal Processing, Vol. 41, no. 6, pp. 2047-2066, Jun. 1993.

[4] S. Wada, "Design of Nonuniform Division Multirate FIR Filter Banks", IEEE Trans. Circuits Syst. II, Vol. 42, no. 2, pp. 115-121, Feb. 1995.

[5] J. Li, T.Q. Nguyen and S. Tantaratana, "A Simple Design Method for Nonuniform Multirate Filter Banks", 28th Asilomar Conf. on signal, systems and computers, Pacific Grove, CA, Oct. 1994, pp. 1015-1019.

[6] J. Princen, "The Design of Nonuniform Modulated Filterbanks", IEEE Trans. Signal Processing, Vol. 43, no. 11, pp. 2550-2560, Nov. 1995.

[7] F. Argenti and E. Del Re, "Non-uniform filter banks based on a multi-prototype cosine modulation", IEEE ICASSP'96, Atlanta, May 1996, pp. 1511-1514.

[8] R.D Koilpillai and P.P. Vaidyanathan, "A Spectral Factorization Approach to Pseudo-QMF Design", IEEE Trans. Signal Processing, Vol. 41, no. 1, pp. 82-92, Jan. 1993.

[9] F. Argenti B. Brogelli and E. Del Re, "Design of filter banks with rational sampling factors based on a multi-prototype cosine modulation", submitted to IEEE Trans. Signal Processing.
Table 2. Results relative to Example 1

\begin{tabular}{|c|c|c|c|}
\hline$N_{0}, N_{1}, N_{2}$ & $N_{3}$ & $E_{p-p}$ & $E_{a, \max }$ \\
\hline 67 & 34 & $6.67 \mathrm{E}-02$ & $3.00 \mathrm{E}-02$ \\
\hline 83 & 42 & $3.06 \mathrm{E}-02$ & $1.20 \mathrm{E}-02$ \\
\hline 123 & 62 & $6.45 \mathrm{E}-03$ & $4.36 \mathrm{E}-03$ \\
\hline 163 & 82 & $4.04 \mathrm{E}-03$ & $9.15 \mathrm{E}-04$ \\
\hline
\end{tabular}

Table 3. Results relative to Example 2

\begin{tabular}{|c|c|c|c|}
\hline$N_{0}, N_{1}, N_{3}$ & $N_{2}$ & $E_{p-p}$ & $E_{a, \max }$ \\
\hline 100 & 298 & $1.26 \mathrm{E}-02$ & $6.55 \mathrm{E}-03$ \\
\hline 170 & 508 & $3.93 \mathrm{E}-03$ & $3.87 \mathrm{E}-03$ \\
\hline
\end{tabular}

[10] R.D Koilpillai and P.P. Vaidyanathan, "CosineModulated FIR Filter Banks Satisfying Perfect Reconstruction", IEEE Trans. Signal Processing, Vol. 40, no. 4, pp. 770-783, Apr. 1992.

[11] T.Q. Nguyen, "Near-Perfect-Reconstruction Pseudo-QMF Banks", IEEE Trans. Signal Processing, Vol. 42, no. 1, pp. 65-76, Jan. 1994.

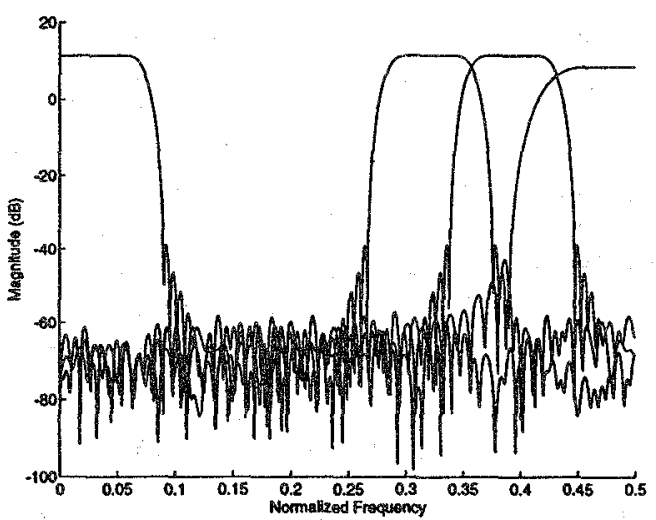

Figure 2. Bank $\{2 / 7,2 / 7,2 / 7,1 / 7\}$

Table 1. Conditions on $\theta_{m}$ for aliasing cancellation

\begin{tabular}{|c|c|c|}
\hline (high)-(high) & $W_{M_{m}}^{\left(k_{m}+1\right) R_{m}}=W_{M_{m+1}}^{ \pm\left(k_{m+1}+1\right) R_{m+1}}$ & $e^{-j 2 \theta_{m}}+e^{\mp j 2 \theta_{m+1}}=0$ \\
\hline (low)-(low) & $W_{M_{m}}^{k_{m} R_{m}}=W_{M_{m+1}}^{ \pm k_{m+1} R_{m+1}}$ & $e^{-j 2 \theta_{m}}+e^{\mp j 2 \theta_{m+1}}=0$ \\
\hline (low)-(high) & $W_{M_{m}}^{k_{m} R_{m}}=W_{M_{m+1}}^{ \pm\left(k_{m+1}+1\right) R_{m+1}}$ & $e^{-j 2 \theta_{m}}+e^{\mp j 2 \theta_{m+1}}=0$ \\
\hline (high)-(low) & $W_{M_{m}}^{\left(k_{m}+1\right) R_{m}}=W_{M_{m+1}}^{ \pm k_{m+1} R_{m+1}}$ & $e^{-j 2 \theta_{m}}+e^{\mp j 2 \theta_{m+1}}=0$ \\
\hline
\end{tabular}

